\title{
El uso periodístico de Facebook y Twitter: un análisis comparativo de la experiencia europea ${ }^{1}$
}

\author{
Sonia GonZÁlez MolinA \\ Universitat Jaume I de Castellón \\ smolina@uji.es \\ Fátima RAMOS DEL CANO \\ Universitat Jaume I de Castellón \\ framos@uji.es
}

\begin{abstract}
Resumen
Actualmente, las redes sociales se configuran como un motor de transformación en las relaciones entre los medios y sus audiencias. Este artículo analiza el uso periodístico de Facebook y Twitter en 41 medios europeos de referencia. La metodología está basada en el análisis de contenido de sus cuentas oficiales. El período de análisis se sitúa en 2013. Los resultados apuntan a una presencia mayoritaria de los medios en Facebook y Twitter y un uso prioritario de difusión de información. Además, es en los países mediterráneos donde se observa una mayor actividad tanto por parte de los medios como de sus usuarios.
\end{abstract}

Palabras Clave: Facebook; Twitter; redes sociales; periodismo; estrategias corporativas; medios europeos

The journalistic use of Facebook and Twitter: a comparative analysis of the European experience

\begin{abstract}
Nowadays, the social networks seem to be the driving force in the transformation of the relationship between the media and their audiences. This paper analyses the journalistic use of Facebook and Twitter in 41 referential European media. The methodology is based on the content analysis of their corporative accounts on 2013. The results show that media are mostly present on Facebook and Twitter and that they mainly use them to distribute information. Above this, the Mediterranean media and audience are those that present more activity in the social networks analysed.
\end{abstract}

Keywords: Facebook, Twitter, Social Networking Service, journalism, corporative strategies, european media

\section{Referencia normalizada:}

González Molina, S.; Ramos del Cano, F. (2013) El uso periodístico de Facebook y Twitter: un análisis comparativo de la experiencia europea. Historia y Comunicación Social. Vol. 18 No Especial Noviembre. Págs. 419-433.

Sumario: 1. Las redes sociales en la esfera periodística. 1.1. Usos y funciones de las redes sociales. 2. Objetivos y metodología. 3. Resultados. 3.1. Facebook y Twitter en los medios. 3.2. Accesibilidad. 3.3. Usos. 3.4. Actividad. 4. Conclusiones. 5. Bibliografía.

1 Esta investación se engloba dentro del proyecto de I+D con referencia P11B2010-53 financiado por la Fundación Caixa Castelló-Bancaixa y la Universitat Jaume I. 


\section{Las redes sociales en la esfera periodística}

La irrupción de las nuevas tecnologías en el mundo de la comunicación está transformado la esfera periodística desde el punto de vista del tránsito informativo (Casero-Ripollés y Feenstra, 2012), del incremento del número de actores participantes en la producción de las noticias (McNair, 2003; Hermida, 2010; Phillips, 2011) y, por último, del de la relación que se establece entre los medios y los ciudadanos.

En este contexto, las redes sociales cobran un creciente protagonismo. En el actual marco europeo, el $42 \%$ de los internautas las utiliza al menos una vez a la semana, y una tercera parte lo hace a diario (TNS-Sofres, 2013). Además, y a pesar de no poder hablar de una situación homogénea en toda Europa, la tónica dominante es de un uso cada vez más habitual. Por países, Letonia (60\%), Países Bajos (60\%), Suecia (59\%), Dinamarca (57\%) y Estonia (53\%), resultan ser los que con más frecuencia emplean las redes semanalmente. Por otro lado, Alemania $(34 \%,+5$ puntos porcentuales), Portugal $(31 \%,+5)$, Rumania $(28 \%,+5)$ y Finlandia $(50 \%,+4)$ son los países con un menor crecimiento del uso de las redes respecto de los últimos tres años. Mientras, los que mayor aumento han experimentado son Estonia $(53 \%,+12)$, Italia $(43 \%$, $+12)$, Letonia $(60 \%,+12)$, Grecia $(39 \%, 13)$, y especialmente España $(48 \%,+16)$ y la República Checa $(39 \%,+16)$.

Del conjunto de redes sociales, las que más usuarios tienen y más han crecido en los últimos diez años son Facebook y Twitter (SilverPop, 2012), objeto de estudio de nuestra investigación. La primera fue creada por Mark Zuckerberg en 2004 como una web para poner en contacto a estudiantes de la Universidad de Harvard, si bien ahora cualquier persona puede formar parte de ella. La segunda entró en funcionamiento en 2006 y permite que cualquier persona $u$ organismo publique y difunda mensajes breves de no más de 140 caracteres. Java et al (2007) indican que, en general, este servicio de microblogging se utiliza para chatear, conversar, compartir información y enviar noticias.

Como el resto de redes sociales, Facebook y Twitter han logrado convertirse en el fenómeno de mayor crecimiento e interés por parte de los internautas al permitir dotarles de la capacidad de lanzar sus mensajes e ideas (Stanyer 2008; Rodrigues, 2010). Ambos servicios pertenecen a la denominada Web 2.0, un concepto que alude a un conjunto de aplicaciones que animan al usuario a participar y colaborar en la creación de contenido. Una posibilidad que dota de valor añadido a unas herramientas que confían plenamente en el uso que les dan sus usuarios (O'Reilly, 2012), tanto desde el punto de vista del empoderamiento (Jenkins, 2006) como de la auto-comunicacion (Castells, 2009).

\subsection{Usos y funciones de las redes sociales}

Las redes sociales están introduciendo una serie de desafíos que tienen que ver con la gestión de la relación que mantienen los medios con su público, un aspecto estratégico en el proceso informativo. Por ello, resulta pertinente examinar el uso que 
les dan a estas nuevas plataformas, cuya irrupción en las redacciones ha comportado también una mayor exigencia en términos de polivalencia profesional para los periodistas (González-Molina y Ortells-Badenes, 2012).

Orihuela (2005) clasifica las redes según su cometido y separa aquellas cuya función es la comunicación, entendida como la puesta en común de conocimientos, de las que se ocupan de crear comunidad y de las que fomentan la cooperación conjunta en la Red. Por su parte, The Cocktail Analysis (2009) distingue entre redes de comunicación inmediata (Twitter o Messenger), comunidades de contenidos (Flickr o Myspace) y redes de exposición (Facebook y Tuenti). Fumero y García (2008) reducen las funciones a únicamente dos, de acuerdo a su carácter social: aquellas que se centran en el contenido producido en comunidad por los usuarios (User Generated Content) y las que lo hacen en la gestión de las relaciones sociales (Online Social Networking).

Sin embargo, la explotación que hacen los medios de su presencia en las redes sociales dista mucho de ser ejemplar. La principal carencia detectada por investigaciones precedentes en este campo es la ausencia de diálogo efectivo con los usuarios, con independencia del medio. En su estudio sobre la prensa inglesa, Hermida y Thurman (2008) detectaron una limitada apertura a la conversación con la audiencia. Un aspecto que Domingo et al (2008) también señalaron con respecto de la europea y estadounidense. Noguera-Vivo (2010) llegó a parecidas conclusiones en su trabajo sobre 13 cabeceras españolas. Más recientemente, García de Torres et al (2011) destacaron la escasa presencia de mensajes conversacionales en los perfiles de Facebook y Twitter de medios españoles e iberoamericanos. La investigación de Ramos del Cano (2013), centrada en este caso en el ámbito radiofónico, abunda en la misma idea. Por último, González Molina (2013) destaca, ya en el entorno del periodismo institucional, la poca orientación dialógica de los organismos.

\section{Objetivos y metodología}

Este artículo busca analizar de qué manera se presentan los medios informativos europeos de referencia en Facebook y Twitter, las dos redes sociales con más número de usuarios y que más crecimiento han experimentado en los últimos diez años (SilverPop, 2012). Este objetivo principal se desglosa en los siguientes:

- Establecer el peso específico de Facebook y Twitter en el conjunto de redes sociales que usan los medios europeos.

- Determinar el grado de accesibilidad a estas cuentas desde las propias páginas web de los medios seleccionados.

- Apuntar el uso prioritario que dan los medios a estos perfiles corporativos.

- Examinar la actividad de los medios en los perfiles y el nivel de respuesta de la audiencia. 
Para ello, se parte de las siguientes hipótesis:

- Facebook y Twitter son las redes sociales prioritarias para los medios europeos.

- Los iconos de acceso a estos perfiles corporativos se encuentran mayoritariamente en la página inicial.

- El uso prioritario que le confieren es el de difundir información.

- La actividad de los medios y los usuarios pertenecientes al modelo comunicativo mediterráneo es la más dinámica.

La metodología escogida para desarrollar la investigación se basa en el análisis del contenido de las cuentas oficiales de Facebook y Twitter de 41 medios europeos de referencia de seis países diferentes, seleccionados en función de su audiencia ${ }^{2}$. Más concretamente, la muestra comprende los dos medios de mayor audiencia en prensa, radio y televisión, y un medio nativo de España, Italia, Alemania, Finlandia ${ }^{3}$, Gran Bretaña y Francia. Esta selección incluye representantes de los tres grandes modelos de sistemas de medios planteados por Hallin y Mancini (2008) tal y como se plasma en la Tabla 1:

Tabla 1: Países analizados según los tres modelos de sistemas de medios.

\begin{tabular}{|c|c|c|}
\hline Modelo & Características del modelo & $\begin{array}{c}\text { Países } \\
\text { seleccionados }\end{array}$ \\
\hline $\begin{array}{c}\text { Mediterráneo } \\
\text { o Pluralista } \\
\text { Polarizado }\end{array}$ & $\begin{array}{c}\text { Se caracteriza por una prensa con tiradas cortas, y } \\
\text { subvencionada. El paralelismo político suele ser alto, ya que } \\
\text { la instrumentalización de los medios por parte del gobierno } \\
\text { es habitual. La profesionalización del periodista no está tan } \\
\text { desarrollada como en los otros modelos. }\end{array}$ & $\begin{array}{c}\text { España, Italia y } \\
\text { Francia }\end{array}$ \\
\hline $\begin{array}{c}\text { Norte y Centro } \\
\text { de Europa o } \\
\begin{array}{c}\text { Democrático } \\
\text { Corporativo }\end{array}\end{array}$ & $\begin{array}{c}\text { Al contrario que el anterior, se caracteriza por un desarrollo } \\
\text { periódicos, con grandes tiradas. El nivel de paralelismo político } \\
\text { es alto, con un alto legado del periodismo de comentario } \\
\text { mezclado con creciente énfasis en una profesionalidad neutral } \\
\text { y un periodismo orientado a la información. El nivel de } \\
\text { profesionalidad del periodista es alto. }\end{array}$ & $\begin{array}{c}\text { Alemania y } \\
\text { Finlandia }\end{array}$ \\
\hline
\end{tabular}

2 Los medios seleccionados son, para España, Telecinco, Televisión Española, Cadena Ser, Onda Cero, El País, El Mundo y Lainformacion.com. En Francia, TF1, France 2, Europe 1, France Bleu, Le Monde, Le Figaro y Rue89. En Italia, Rai 1, Canale 5, RaiRadio1, Radio Deejay, Il Corriere de la Sera, La Repubblica y Libero Nato Digitale. En Finlandia, Yle TV2, MTV3, YLE Radio Suomi, Helsingin Sanomat, Aamulehti y Digitoday. En Gran Bretaña, BBC1, ITV, BBC Radio 2, BBC Radio 4, Telegraph, The Times y Hackney Citizen. En Alemania, RTL Television, ErstesDeutschesFernsehen, Antenne Bayern, Hit-Radio Antenne, SüddeutscheZeitung, Frankfurter Allgemeine y Perlentaucher.

3 En el caso de Finlandia, la única emisora de radio de cobertura nacional es YLE por lo que solamente se ha incluido un medio radiofónico en la muestra. 


\begin{tabular}{|c|c|c|}
\hline $\begin{array}{c}\text { Atlántico Norte } \\
\text { o Liberal }\end{array}$ & $\begin{array}{l}\text { Igual que el anterior, se caracteriza por un desarrollo temprano } \\
\text { de la libertad de prensa y por una prensa de gran tirada. } \\
\text { En este sistema, domina la prensa comercial, el nivel de } \\
\text { paralelismo político es bajo y predomina el pluralismo interno. } \\
\text { El periodismo se orienta hacia la información, con unos } \\
\text { profesionales bien organizados profesionalmente y bastante } \\
\text { aislados de un posible control político. }\end{array}$ & Gran Bretaña \\
\hline
\end{tabular}

Fuente: elaboración propia.

El trabajo de campo se desarrolló durante los meses de mayo a septiembre, en los que se escogieron diversos días al azar (laborables y festivos) para examinar los mensajes publicados en las cuentas corporativas de los medios en Facebook y Twitter. Para analizarlos, se elaboró una ficha que contempla los aspectos recogidos en la Tabla 2.

Tabla 2: Dimensiones y categorías de análisis de la presencia en redes sociales.

\begin{tabular}{|c|c|c|c|}
\hline Dimensiones & & Categorías & Explicación \\
\hline \multirow{2}{*}{$\begin{array}{l}\text { Presencia } \\
\text { pública }\end{array}$} & \multicolumn{2}{|c|}{ Medios encontrados } & $\begin{array}{c}\text { Enumeración de las redes sociales encontradas y } \\
\text { situación (home o interior) }\end{array}$ \\
\hline & \multicolumn{2}{|r|}{ Accesibilidad } & Situación: home (arriba, abajo, al lado) y/o interior \\
\hline \multirow{4}{*}{ Función } & \multicolumn{2}{|r|}{ Promoción } & $\begin{array}{l}\text { Se da publicidad de otro medio del grupo o de } \\
\text { programas }\end{array}$ \\
\hline & \multicolumn{2}{|r|}{ Información } & $\begin{array}{l}\text { Se facilita información sobre algo, como titulares, } \\
\text { avances, últimas horas, etc. }\end{array}$ \\
\hline & \multicolumn{2}{|r|}{ Diálogo } & $\begin{array}{l}\text { Se interpela al usuario en forma de preguntas directas } \\
\text { en encuestas, foros, etc. }\end{array}$ \\
\hline & \multicolumn{2}{|r|}{ Usuario } & $\begin{array}{l}\text { Se pide al usuario que facilite más información o } \\
\text { detalles sobre algún suceso. }\end{array}$ \\
\hline \multirow{8}{*}{ Actividad } & \multirow{4}{*}{ 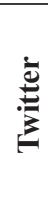 } & Followers & Número de seguidores \\
\hline & & Following & Número de usuarios a los que sigue el medio \\
\hline & & Retuits & Número de veces que el tuit se ha redifundido \\
\hline & & Favoritos & $\begin{array}{l}\text { Número de veces en que el tuit se ha marcado como } \\
\text { favorito }\end{array}$ \\
\hline & \multirow{4}{*}{ 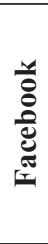 } & Me gusta & $\begin{array}{l}\text { Número de veces que la página de Facebook ha sido } \\
\text { marcada así }\end{array}$ \\
\hline & & Me gusta en post & Número de veces que el post ha sido marcado así \\
\hline & & $\begin{array}{c}\text { Páginas que le } \\
\text { gustan al medio }\end{array}$ & Número de páginas que le gustan al medio \\
\hline & & Compartir & Número de veces que el post se ha compartido \\
\hline
\end{tabular}

Fuente: elaboración propia. 


\section{Resultados}

A continuación, exponemos los resultados más significativos del trabajo de campo:

\subsection{Facebook y Twitter en los medios}

Facebook y Twitter, con escasa diferencia entre ambas, son las redes sociales con un uso más extendido entre los medios de comunicación europeos, como se puede observar en la Tabla 3, en la que se contabilizan las cabeceras en función de los perfiles encontrados. De hecho, estas dos opciones constituyen el 51,64\% de los resultados analizados (27,15\% para Facebook y 24,49\% para Twitter). Muy cerca, les sigue Google + $(19,86 \%)$ y, a más distancia, opciones como Tuenti o Menéame $(3,9 \%)$, sólo presentes en los medios españoles.

Tabla 3: Número de medios europeos con perfiles en redes sociales.

\begin{tabular}{|l|c|c|c|c|c|c|}
\hline & Facebook & Twitter & Google + & Linkedin & Tuenti & Menéame \\
\hline España & 7 & 7 & 6 & 2 & 6 & 6 \\
\hline Italia & 7 & 6 & 5 & 1 & 0 & 0 \\
\hline Francia & 7 & 7 & 6 & 2 & 0 & 0 \\
\hline Alemania & 7 & 7 & 6 & 0 & 0 & 0 \\
\hline Finlandia & 6 & 6 & 4 & 1 & 0 & 0 \\
\hline Gran Bretaña & 7 & 7 & 3 & 2 & 0 & 0 \\
\hline TOTAL & $\mathbf{4 1}$ & $\mathbf{4 0}$ & $\mathbf{3 0}$ & $\mathbf{8}$ & $\mathbf{6}$ & $\mathbf{6}$ \\
\hline
\end{tabular}

Fuente: elaboración propia.

En el trabajo de campo, también se ha detectado otras opciones minoritarias (la Tabla 3 sólo refleja las seis plataformas más habituales), propias de cada país. Este es el caso de StumbleUpon (Gran Bretaña), Dailymotion (Francia) o StudiVZ (Alemania). Igualmente, ha permitido comprobar que algunos medios han optado por estar presentes únicamente en una única red social, como es el caso de Canale 5 y Facebook. Por el contrario, otras cabeceras destacan por su abundante oferta, agrupadas en una pestaña específica. Este es el caso de las italianas Rai Radio 1 y Rai 1 (más de 100 plataformas), y las inglesas ITV Televisión (más de 300) o la radio francesa France Bleu (ver Imagen 1). 
Imagen 1. Captura de pantalla de la radio France Bleu

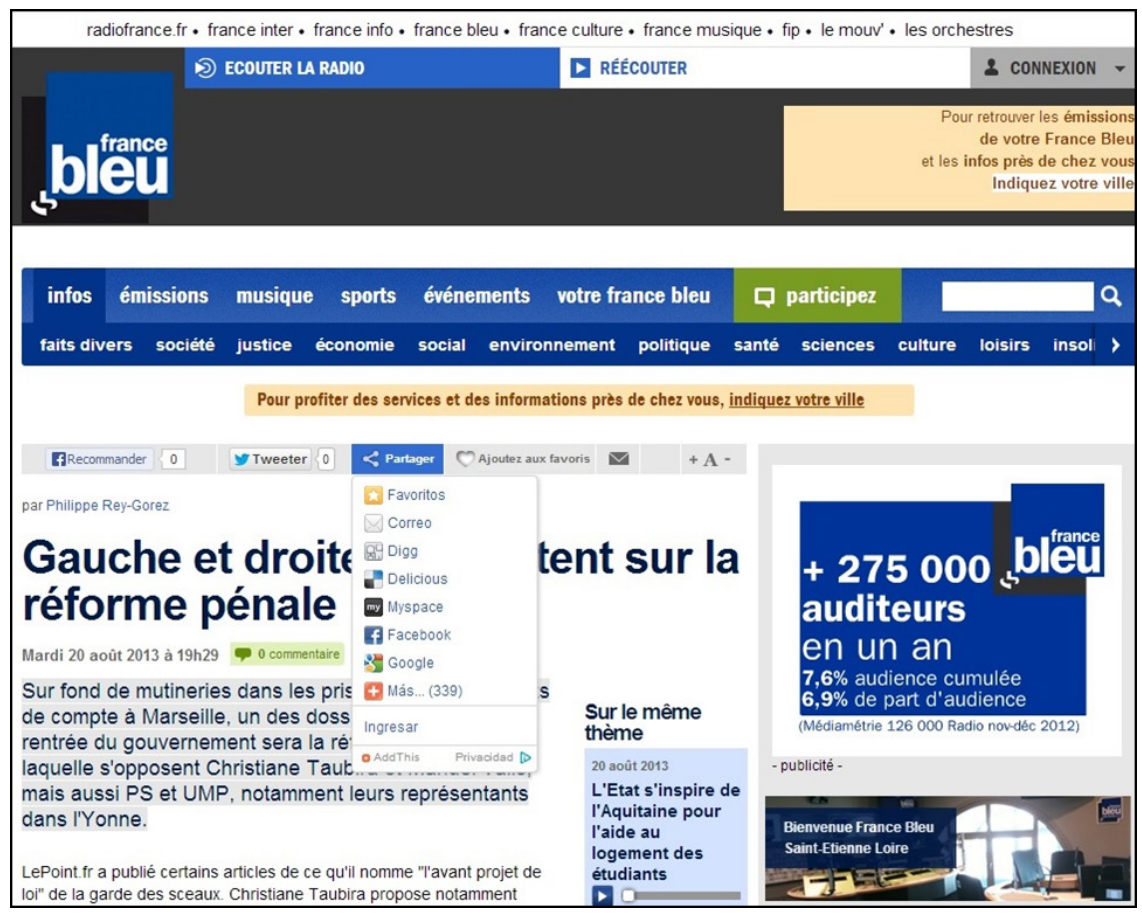

\subsection{Accesibilidad}

No todos los medios analizados publicitan los iconos de acceso a los perfiles corporativos de Facebook y Twitter desde la home aunque sí desde las páginas interiores, es decir, dentro de cada noticia. Como se puede comprobar en la Tabla 4, la diferencia sin ser excesiva, es significativa $(78,04 \%$ frente al $100 \%)$ :

Tabla 4: Situación de los iconos de acceso a Facebook y Twitter.

\begin{tabular}{|l|c|c|c|c|c|}
\hline & \multicolumn{2}{|l|}{ Situación } & \multicolumn{3}{l|}{ Situación en home } \\
\hline & Home & Interior & Arriba & Abajo & Al lado \\
\hline España & 7 & 7 & 6 & 0 & 4 \\
\hline Italia & 6 & 7 & 5 & 2 & 2 \\
\hline Francia & 7 & 7 & 6 & 5 & 3 \\
\hline Alemania & 5 & 7 & 2 & 1 & 5 \\
\hline Finlandia & 2 & 6 & 0 & 0 & 2 \\
\hline Gran Bretaña & 5 & 7 & 0 & 4 & 2 \\
\hline TOTAL & $\mathbf{3 2}$ & $\mathbf{4 1}$ & $\mathbf{1 9}$ & $\mathbf{1 2}$ & $\mathbf{1 8}$ \\
\hline
\end{tabular}

Fuente: elaboración propia 
La parte superior de la página inicial, donde la visibilidad es mayor (Salaverría y Sancho, 2007), es la ubicación elegida mayoritariamente por los medios para situar sus iconos en la página inicial. Otra fórmula habitualmente usada es el lateral, mientras que la parte inferior es la opción menos frecuente. Sólo tres de los 41 medios analizados (Le monde, Europe 1 y Rue 89, todos franceses) cuentan con un icono en los tres sitios. La mayoría medios optan por una doble ubicación.

Por países, los resultados del trabajo de campo son bastante homogéneos con la excepción de Finlandia, con sólo dos de las seis cabeceras analizadas con iconos en la página inicial, siempre en la parte inferior. Uno de estos medios, Digitoday, tiene un enlace en su home pero al perfil de Twitter de Taloussanomat, una publicación digital del mismo grupo (Sanomat News).

\subsection{Usos}

En términos generales, el uso más frecuente que los medios europeos dan a sus perfiles en Facebook y Twitter es el de difundir información (ver Tabla 5). Así, de los 2.274 mensajes analizados, un $81,09 \%$ se encuadra en esta categoría, mientras que un $13,76 \%$ pertenece a la de promoción y un $5,10 \%$ a la de diálogo con el usuario. Un marginal $0,05 \%$ se corresponde a los mensajes en los que el medio usa al público como fuente informativa.

Tabla 5: Usos de Facebook y Twitter por los medios europeos.

\begin{tabular}{|c|c|c|c|c|c|c|c|c|}
\hline & \multicolumn{2}{|c|}{ Promoción } & \multicolumn{2}{c|}{ Información } & \multicolumn{2}{c|}{ Diálogo } & \multicolumn{2}{c|}{ Usuario } \\
\hline & FB & TW & FB & TW & FB & TW & FB & TW \\
\hline España & 20 & 37 & 109 & 245 & 24 & 2 & 1 & 0 \\
\hline Italia & 9 & 9 & 126 & 472 & 6 & 2 & 0 & 0 \\
\hline Francia & 5 & 33 & 102 & 274 & 8 & 2 & 0 & 0 \\
\hline Alemania & 20 & 18 & 26 & 127 & 4 & 5 & 0 & 0 \\
\hline Finlandia & 6 & 9 & 11 & 8 & 4 & 0 & 0 & 0 \\
\hline Gran Bretaña & 58 & 89 & 24 & 320 & 18 & 36 & 0 & 0 \\
\hline TOTAL FB/TW & $\mathbf{1 1 8}$ & $\mathbf{1 9 5}$ & $\mathbf{3 9 8}$ & $\mathbf{1 4 4 6}$ & $\mathbf{6 4}$ & $\mathbf{5 2}$ & $\mathbf{1}$ & $\mathbf{0}$ \\
\hline TOTAL GRAL & \multicolumn{3}{|c|}{$\mathbf{3 1 3}$} & \multicolumn{7}{|c|}{$\mathbf{1 8 4 4}$} & $\mathbf{1 1 6}$ & \multicolumn{1}{|c}{} \\
\hline
\end{tabular}

Fuente: elaboración propia.

El uso informativo también es el predominante tanto en Facebook como en Twitter si se analizan de manera separada (68,50\% y $84,41 \%$, respectivamente). Con todo, se aprecia una tendencia más significativa en Facebook hacia los post promocionales y los dialogantes: $68,50 \%$ y $11,05 \%$ frente al $11,51 \%$ y $3,07 \%$ de Twitter.

Por países, los medios que hacen un uso más intensivo de Facebook como mecanismo promocional son los ingleses. Aquí, se han contabilizado 58 mensajes de la categoría de promoción frente a los 24 de la informativa. Por otro lado, los alemanes 
también parecen apostar por esta plataforma con idénticos fines, aunque sin llegar a desbancar al uso informativo ( 20 mensajes promocionales frente a 26 informativos).

Respecto al servicio de microblogging Twitter, la tendencia dominante es el de utilizarlo para informar a la audiencia, con la única excepción de Finlandia en que los usos informativos y promocionales están prácticamente empatados (8 y 9 tuits, respectivamente).

El trabajo de campo también permite detectar el escaso uso dialógico que hacen los medios de ambos servicios. Partiendo de esta base, podemos destacar que el país más dialogante en Facebook es España y el segundo, Gran Bretaña. A su vez, este último obtiene más tuits dentro de esta categoría, seguido por Alemania. De hecho, el único representante del modelo liberal es el que presenta más mensajes dentro de la dimensión vinculada al diálogo: 54, ligeramente más que el doble que los españoles (26). Hay que reseñar, en este sentido, que los medios ingleses utilizan estas plataformas en mayor medida que el resto de cabeceras para hacer entrevistas en línea animando al público a plantear preguntas o pidiéndoles expresamente su opinión en relación a algún asunto.

\subsection{Actividad}

En relación a la actividad de los medios en Facebook, destacaremos en primer lugar que unos once millones de europeos siguen a alguna cabecera de las seleccionadas, tal y como se refleja en el apartado "Me Gusta la página" de la Tabla 6. Sobresalen los medios franceses, que aportan un 36,4\% de seguidores, seguidos de los italianos $(28,5 \%)$ y los españoles $(14,1 \%)$, todos ellos pertenecientes a países de modelo mediterráneo. Finlandia destaca por ser el que menos aporta, con un anecdótico $1,2 \%$ del total.

Si miramos el número de perfiles que los medios siguen desde su cuenta en Facebook, son los británicos los que se muestran más inclinados a esta práctica, ya que el 44,1\% del total de "Páginas que les gustan al medio" es suyo. A continuación, se sitúan los medios franceses $(19,7 \%)$ y los españoles $(15,1 \%)$. Los medios finlandeses y alemanes son los menos abiertos, con un 6,3 y un $4,8 \%$, respectivamente.

Los medios finlandeses también destacan por ser los que menos actividad propia registran en sus perfiles de Facebook, ya que sólo un 3,2\% de los 579 mensajes analizados durante el trabajo de campo son suyos. Los medios españoles, seguidos de los italianos, son los que registraron una mayor actividad con un 26,5 y un $24,3 \%$, respectivamente del total de post publicados, revelando un uso más intensivo de este servicio. 
Tabla 6. Actividad de los medios europeos en Facebook.

\begin{tabular}{|c|c|c|c|c|c|}
\hline FACEBOOK & $\begin{array}{c}\text { Número de } \\
\text { publicaciones* }^{*}\end{array}$ & $\begin{array}{c}\text { Me Gusta la } \\
\text { Página*** }^{*}\end{array}$ & $\begin{array}{c}\text { Páginas que le } \\
\text { gustan al medio }\end{array}$ & $\begin{array}{c}\text { Me gusta } \\
\text { el Post }\end{array}$ & Compartir \\
\hline España & 154 & 1.564 .474 & 276 & 32.265 & 7.446 \\
\hline Italia & 141 & 3.158 .807 & 180 & 66.546 & 26.703 \\
\hline Francia & 115 & 4.031 .516 & 360 & 32.476 & 12.796 \\
\hline Alemania & 50 & 1.163 .055 & 88 & 4.336 & 1.253 \\
\hline Finlandia & 19 & 141.670 & 115 & 895 & 118 \\
\hline Gran Bretaña & 100 & 1.005 .306 & 805 & 17.188 & 2.820 \\
\hline TOTAL & $\mathbf{5 7 9}$ & $\mathbf{1 1 . 0 6 4 . 8 2 8}$ & $\mathbf{1 . 8 2 4}$ & $\mathbf{1 5 3 . 7 0 6}$ & $\mathbf{5 1 . 1 3 6}$ \\
\hline
\end{tabular}

* Número de post publicados durante los días en los que se desarrolló el trabajo de campo ** Número de seguidores del perfil corporativo

Fuente: elaboración propia.

En relación a la actividad de la audiencia, los usuarios italianos son los que mayor acogida muestran a los mensajes en Facebook de sus medios (expresada con los "me gusta" en los post), con un sobresaliente $43,2 \%$, muy superior al $21,1 \%$ de los franceses y al $20,9 \%$ de los españoles. Algunos medios son especialmente efectivos, ya que con única publicación al día pueden llegar a obtener más de dos mil "Me gusta" como se observa en la Imagen 2. La audiencia italiana es también la más activa compartiendo contenidos, con un 52,2\% del total de mensajes compartidos. Por el contrario, la finlandesa es la menos animada con sólo un $0,2 \%$ del total.

Imagen 2. Captura de pantalla del Facebook de la francesa TF1

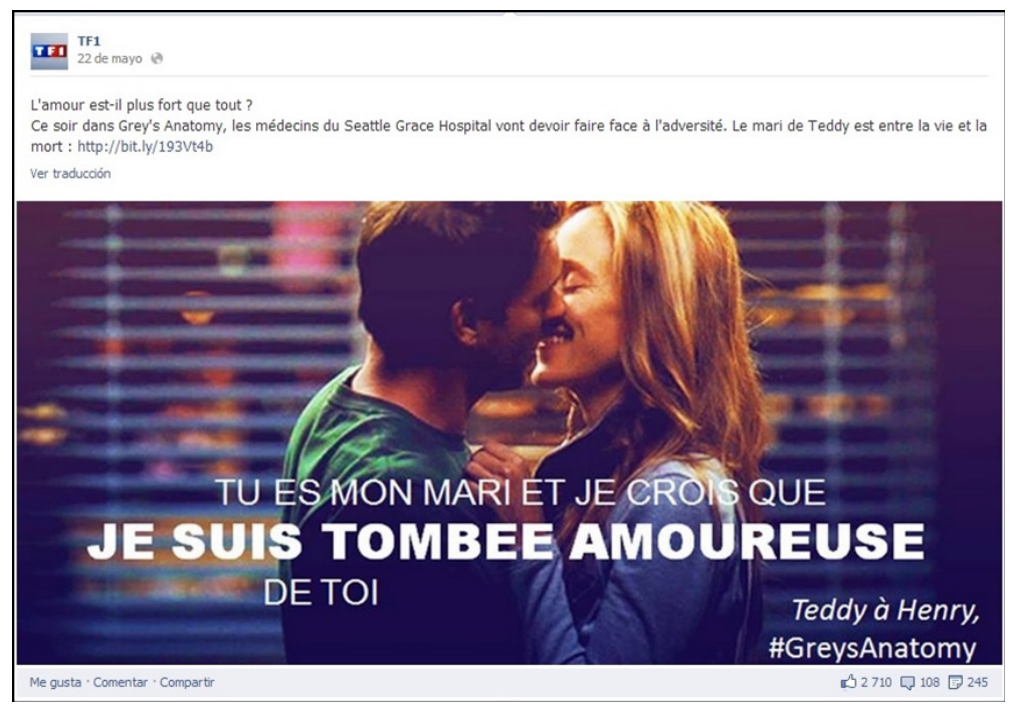


Los resultados en Twitter son similares a los ya explicados en Facebook, como se puede observar en la Tabla 7 . Atendiendo al número de seguidores, Francia $(37 \%$ del total de followers), España $(25,2 \%)$ e Italia $(21,4 \%)$ ocupan las primeras posiciones mientras que Finlandia se sitúa en el último lugar $(0,6 \%)$. Los países del modelo mediterráneo copan el 83,7\% del total de la muestra. Sin embargo, es Gran Bretaña la que destaca en cuánto a otras cuentas seguidas por sus medios: los ingleses son los que más proclives se muestran a seguir otros perfiles de Twitter, como lo demuestra el $64,1 \%$ del total. Los medios españoles, sin embargo, son los que menos lo hacen $(1,1 \%)$.

Tabla 7. Actividad de los medios europeos en Twitter.

\begin{tabular}{|c|c|c|c|c|c|c|}
\hline TWITTER & $\begin{array}{c}\text { Número de } \\
\text { Tuits } \\
\text { TOTAL* }\end{array}$ & $\begin{array}{c}\text { Número de } \\
\text { Tuits } \\
\text { Enviados** }\end{array}$ & Followers & Following & Retuits & Favoritos \\
\hline España & 117.066 & 62 & 2.935 .340 & 489 & 5.786 & 1.177 \\
\hline Italia & 170.225 & 483 & 2.477 .432 & 1.280 & 3.965 & 1.272 \\
\hline Francia & 293.106 & 309 & 4.280 .090 & 3.118 & 4.637 & 1.130 \\
\hline Alemania & 162.659 & 148 & 424.326 & 5.003 & 519 & 170 \\
\hline Finlandia & 87.408 & 23 & 76.924 & 5.198 & 120 & 9 \\
\hline Gran Bretaña & 324.361 & 445 & 1.366 .979 & 27.014 & 4.859 & 1.181 \\
\hline TOTAL & $\mathbf{1 . 1 5 4 . 8 2 5}$ & $\mathbf{1 . 4 7 0}$ & $\mathbf{1 1 . 5 6 1 . 0 9 1}$ & $\mathbf{4 2 . 1 0 2}$ & $\mathbf{1 9 . 8 8 6}$ & $\mathbf{4 . 9 3 9}$ \\
\hline
\end{tabular}

* Número de Tutis publicados desde el inicio de la actividad del medio en Twitter a fecha de 18 de septiembre de 2013

** Número de Tuits publicados durante los días en los que se desarrolló el trabajo de campo

Fuente: elaboración propia.

Durante el trabajo de campo, se publicaron unos 1.400 tuits, de los que el 32.8\% pertenecen a medios italianos. No obstante, los medios que más actividad han registrado en relación al volumen de mensajes publicados desde su incorporación a Twitter son los ingleses (el 28\% del millón de tuits detectado). Finlandia se mantiene como el país con menos actividad social, con un $7,5 \%$ en cuanto al total de publicaciones y un $1,5 \%$ de tuits durante el trabajo de campo. España destaca también por el escaso volumen de mensajes, sobre todo si se tienen en cuenta los resultados en Facebook.

En relación a la actividad de la audiencia, los medios españoles, franceses e italianos tienen las más dinámicas. Los usuarios españoles son los que más retuitean (29\%), seguidos por los franceses $(23,3 \%)$ y los italianos (19,9\%). Italia es, además, el país cuyos usuarios han marcado más veces como favoritas las publicaciones de sus medios de origen (25,7\%), seguido de Gran Bretaña (23,9\%). Finlandia destaca en este apartado por su $0,1 \%$, lo que pone de relieve su anecdótica actividad en las redes. 


\section{Conclusiones}

Tras analizar cómo utilizan los medios europeos de referencia de sus perfiles corporativos en Facebook y Twitter respecto a la accesibilidad, uso y actividad, podemos concluir que:

- Facebook y Twitter son las dos redes sociales en las que mayor presencia tienen los medios europeos analizados, tal y como avanzamos en la primera hipótesis. Todos tienen un perfil en Facebook y todos menos uno disponen de una cuenta en Twitter, mientras que Google + es la tercera red social en importancia. No se aprecian diferencias entre los países analizados, más allá de la aparición de servicios propios de los territorios concretos. Con todo, algunos medios destacan por su abundante oferta frente a otros, con una de carácter más bien testimonial.

- Los iconos de acceso a estos perfiles corporativos se encuentran mayoritariamente en las páginas interiores y no en la principal como suponíamos en nuestra segunda hipótesis. Así, existen medios que no sitúan los iconos de acceso en las home; sin embargo, todos ellos los incluyen en las noticias interiores. Tampoco existirían diferencias significativas en cuanto a los países, con la única excepción de Finlandia, el que más claramente opta por no dar visibilidad a sus iconos de acceso desde la portada.

- El uso prioritario que confieren los medios a Facebook y Twitter es el de difundir información, como se recogió en la tercera hipótesis. El segundo es el promocional, si bien se sitúa a bastante distancia. Por plataformas, se observa una tendencia a destinar Twitter a un uso claramente informativo mientras que Facebook se percibe como una herramienta más útil para la promoción. A pesar de la naturaleza dialógica de estas herramientas, la conversación con el público no se configura como un uso prioritario, más bien al contrario. Teniendo esto en cuenta, un país destaca sobre el resto en relación a este uso: Gran Bretaña, con más del doble de mensajes en los que interpela al público o conversa con él que el segundo de la lista, España.

- Los países pertenecientes al modelo mediterráneo han destacado muy por encima de los restantes analizados tanto en la actividad que sus medios en sus perfiles sociales como en el nivel de respuesta que han logrado por parte de sus usuarios. En el otro lado, encontramos a Finlandia (representante del modelo del Norte y Centro de Europa) como el que menor uso le ha conferido a sus herramientas sociales, obteniendo por parte de su audiencia una respuesta acorde a esta actitud.

Para acabar, diremos que esta investigación, de naturaleza eminentemente cuantitativa, se ha centrado únicamente en una comparativa entre países y no entre medios. No obstante, las diferencias más significativas parecen darse más bien entre cabeceras, tal y como se intuye en el trabajo de campo. Por ello, de cara al futuro, sería acon- 
sejable completar la propuesta contemplando esta posibilidad, añadiendo también un análisis cualitativo de los datos.

\section{Bibliografía}

CASERO-RIPOLLÉS, A. y FEENSTRA, R. A. (2012). "The 15-M Movement and the new media: A case study of how new themes were introduced into Spanish political discourse". En: MIA. Media International Australia, n¹44, pp. 68-76.

CASTELLS, M. (2009). Communication Power. Oxford: Oxford University Press.

DOMINGO, D. et al. (2008). "Participatory journalism practices in the media and beyond: An international comparative study of initiatives in online newspapers".

En: Journalism practice, vol. 2, n³, pp. 326-342.

FUMERO, A. ; GARCÍA, M. (2008): Redes sociales. Contextualización de un fenómeno 'dos-punto-cero'. En: Revista Telos, 76. Disponible en: http://sociedadinformacion.fundacion.telefonica.com/telos/articulocuaderno.asp@idarticu$1 \mathrm{o}=3 \& \mathrm{rev}=76 . \mathrm{htm}[20-09-2013]$.

GARCÍA DE TORRES, E. et al. (2011). "Uso de Twitter y Facebook por los medios iberoamericanos". En: El profesional de la información, vol. 20, n6, pp. 611-620.

GONZÁLEZ MOLINA, S. (2013). "El uso de Twitter en el entorno del Periodismo institucional 2.0: estrategias cross-media y diálogo informativo". En: Icono 14, vol. 11, no 2, pp. 141-162.

GONZÁLEZ MOLINA, S.; ORTELLS BADENES, S. (2012). "La polivalencia periodística de los profesionales en las redes sociales". En: Estudios sobre el mensaje periodístico, vol. 18, pp. 455-463.

HALLIN, C. D. y MANCINI, P. (2008). Sistemas mediáticos comparados. Tres modelos de relación entre los medios de comunicación y la política. Barcelona: Hacer.

HERMIDA, A. (2010). "Twittering the News. The emergence of ambient journalism”. En: Journalism Practice, vol.4, no 3, pág. 297-308.

HERMIDA, A.; THURMAN, N. (2008). "A Clash of Cultures. The integration of user-generated content within professional journalistic frameworks at British newspaper websites". En: Journalism practice, vol. 2, n 3, pp. 343-56.

JAVA, A. et al (2007). "Why we twitter: understanding microblogging usage and communities". En: Proceedings of the 9th WebKDD and 1st SNA-KDD 2007 workshop on Web mining and social network analysis . ACM, 2007. p. 56-65.

JENKINS, H. (2006). Convergence Culture. Where old and new media collide. Nueva York: New York Press.

MCNAIR, B. (2003). "From Control to Chaos. Towards a New Sociology of Journalism”. En: Media, Culture and Society. 2003, vol. 25, n 4, pp. 547-555.

NOGUERA-VIVO, J. M. (2010). "Redes sociales como paradigma periodístico. Medios españoles en Facebook". En: Revista latina de comunicación social. Disponible en: http:/www.revistalatinacs.org/10/art/891_UCAM/13_JM Noguera.html [15-05-2013]. 
O'REILLY, T. (2012). "What is Web 2.0? Design Patterns and Business Models for the Next Generation of Software". En: MANDIBERG, M (2001). The Social Media Reader. New York-London. New York University Press, pp. 32-52.

ORIHUELA, J. L. (2005). “Apuntes sobre redes sociales". En: eCuaderno.com. Disponible en: http://www.ecuaderno.com/2005/07/19/apuntes-sobre-redes-sociales/ [25-07-2013].

PHILLIPS, A. (2011). "Faster and shallower: homogenization, cannibalization and the death of reporting". En LEE, W. P.; PHILLIPS, A. y WITSCHGE, T. (2011). Changing journalism. Londres: Routledge, pp. 81-98.

RAMOS DEL CANO, F., (2013). " La Cadena SER en Facebook: nuevas estrategias de interacción con la audiencia". En: Fonseca, Journal of Communication, nº, pp. 111-134.

RODRÍGUES, C. (2010). "Redes Sociais e práticas que se impõem ao jornalismo". En: II Congreso Internacional Comunicación 3.0, Universidad de Salamanca. [28-08-2013].

SALAVERRÍA, R; SANCHO, F. (2007). "Del papel a la Web: Evolución y claves del diseño periodístico en Internet". En: LARRONDO, A. y SERRANO, A. Diseño periodístico en internet. Leioa: Servicio Editorial de la Universidad del País Vasco, pp. 207-239.

STANYER, J. (2008). "Web 2.0 and the transformation of news and journalism". En: CHADWICK A. y HOWARD, P. N. (eds.) Routledge Handbook of Internet Politics. Londres: Routledge, pp. 201-13.

SILVERPOP, (2012). "Infographic: The Social Network Landscape" En: SilverPop. Disponible en: http://www.silverpop.com/blogs/email-marketing/social-network-growth-infographic.html [05-09-2013].

THE COCKTAILANALYSIS (2009): "I Oleada del Observatorio de Redes Sociales". En: Tcanalysis. Disponible en: http://tcanalysis.com/blog/posts/informe-ebooks. [02-05-2013].

TSN-SOFRES. (2013). “Think. Insights for European growth”. En: Tns-Sofres. Disponible en: http://www.tnsglobal.es/ [14-08-2013].

\section{Las autoras}

Sonia González Molina es profesora ayudante del Departamento de Ciencias de la Comunicación de la Universitat Jaume I (UJI) de Castellón. Es doctora en Comunicación y Humanidades por la Universitat Ramon Llull de Barcelona. Sus principales líneas de investigación se centran en el Ciberperiodismo, el Periodismo institucional y el Periodismo de servicio.

Fátima Ramos del Cano es profesora del Departamento de Ciencias de la Comunicación, en la Universitat Jaume I de Castellón (UJI). Es licenciada en Periodismo 
por la Universidad Carlos III de Madrid y en Historia por la Universidad de León. Su línea de investigación está centrada en el ámbito radiofónico y las implicaciones que el fenómeno de las redes sociales está teniendo en el mismo. 